\title{
Consumo de drogas de diseño entre los adolescentes españoles: Determinantes demográficos y psicosociales
}

\author{
Marta Gil Lacruz, Universidad de Zaragoza (España); \\ Ana Isabel Gil Lacruz, Universidad Autónoma de Madrid (España) \\ Recibido: 6 de mayo del 2009 / Aprobado: 4 de julio del 2009
}

En este artículo analizamos los determinantes del consumo de drogas de diseño entre estudiantes de 14 a 18 años. El trabajo lo concretamos en la sociedad española con la Encuesta sobre Drogas a la Población Escolar (1994-2006). Nuestros resultados evidencian que el consumo de estas sustancias no se reduce. Los jóvenes demandan las sustancias sintéticas como una alternativa de ocio, aunque son conscientes de que su consumo conlleva problemas de salud y sociales. Las diferencias de género revelan que los varones son más propensos a consumir drogas de diseño, y según las variables económicas la capacidad adquisitiva está positivamente correlacionada con la demanda.

drogas de diseño / escolares / entorno social

Club drug consumption among Spanish adolescents: sociodemographic and psychosocial determinants

The main goal of this article is to analyze the determinants of club drugs among Spanish high-school students from 14 to 18 years old. We have drawn data from the Spanish National School Population Survey on Drug Use (1994-2006). There is empirical evidence that club drug consumption among school students remains stable and that students consume them to have fun although they know they cause health and social problems. Gender differences reveal that male students are more likely to consume club drugs than their female counterparts and economic variables reveal that the student's acquisition power is positively correlated with the club drugs' demand.

club drugs / school students / social environment

Correos electrónicos: mglacruz@unizar.es; ana.gil@uam.es 


\section{INTRODUCCIÓN}

Las drogas de diseño son sustancias producidas por síntesis química en laboratorios clandestinos. Generalmente, se trata de compuestos de anfetaminas a los que se añade algún componente de efecto más o menos alucinógeno. Se comercializan en forma de pastillas o comprimidos, y sobre su superficie se graban diversos dibujos que sirven para su identificación. La más conocida es el éxtasis (Plan Nacional sobre Drogas, 2001).

Al principio se consideraban sustancias inocuas, y no se les dedicó la suficiente atención en comparación con la investigación global en drogodependencias, y menos aún estudios experimentales y epidemiológicos (Aleixandre, Valderrama \& Cervera, 2000). Desde los años noventa, el estudio de estos temas en España ha aumentado considerablemente, permitiendo la detección de cambios en las formas y niveles de consumo (Buela-Casal \& Sierra, 2001; Pérez et al., 2007).

Actualmente, los resultados de estas investigaciones alertan seriamente sobre los efectos de la adicción a drogas de diseño. De su consumo se derivan efectos psicológicos (sociabilidad, empatía, euforia, sensación de autoestima aumentada, desinhibición, deseo sexual aumentado, locuacidad, inquietud, confusión, agobio) y efectos fisiológicos (taquicardia, arritmia, hipertensión, sequedad de la boca, sudoración, contrac- ción de la mandíbula, temblores, deshidratación, aumento de la temperatura corporal). Pero también conllevan serios riesgos de trastornos psicológicos (crisis de ansiedad, trastornos depresivos, alteraciones psicóticas) y fisiológicos (aumento severo de la temperatura corporal, arritmia, convulsiones, insuficiencia renal, coagulopatía, hemorragias, trombosis, infartos cerebrales, insuficiencia hepática) (Hussey \& Singer, 1997; National Institute on Drug Abuse, 2001; Pruitt, 1999; U.S Department of Health and Human Services, 2003).

En este artículo nos centramos en el consumo de drogas sintéticas por parte de los jóvenes por tres motivos principales. En primer lugar, los patrones de consumo de psicoactivos se consolidan por lo general en la adolescencia. En segundo lugar, la adolescencia representa uno de los grupos poblacionales más vulnerables, al tener un menor grado de tolerancia a los efectos de las drogas. En tercer lugar, los jóvenes disponen por lo general de presupuestos limitados que pueden conducir a demandar sustancias de peor calidad o más perniciosas.

En el consumo y abuso de estas sustancias se percibe la importancia que el entorno psico-socioeconómico juega en los determinantes de la demanda de las drogas sintéticas en la adolescencia. La lectura multidisciplinar resulta obligada y exige tener en cuenta la subjetividad y la percepción del propio adolescente. Como plantean Fernández et al. (2003), es de gran interés analizar 
los aspectos relacionados con el uso de las drogas desde la perspectiva de los patrones de consumo de estas sustancias, y también las motivaciones para su uso, los efectos que buscan los jóvenes, la percepción de los riesgos y daños que puede causar dicho consumo, así como la actitud hacia estas y su uso. Con este conocimiento estaríamos en condiciones de planificar e implementar pautas ajustadas de prevención y reducción de riesgos.

El resto del artículo se estructura de la siguiente manera. En la segunda sección se revisa la literatura científica sobre el tema, y en la tercera se describe la base de datos y unos primeros estadísticos descriptivos. La cuarta sección aborda los determinantes psicosociodemográficos y la quinta incluye el análisis de resultados y las conclusiones, así como algunas recomendaciones al respecto.

\section{REVISIÓN DE LA LITERATURA CIENTÍFICA}

El número de consumidores de drogas ilegales en el mundo era superior a 185 millones de personas en el 2004. Solo un año más tarde, la cifra había ascendido a 200 millones de personas, es decir un $5 \%$ de la población mundial entre 15 y 64 años. Este 5\% poblacional se distribuía el consumo de la siguiente manera: marihuana (4\%), anfetaminas $(0,6 \%)$, opiáceos $(0,4 \%)$, cocaína $(0,3 \%)$ y éxtasis $(0,2 \%)$. En estas cifras, las drogas de diseño suponen aproximadamente un quinto del total del consumo adictivo (Informe Mundial de Drogas de la Oficina contra la Droga y el Crimen de la Organización de las Naciones Unidas (UNODC, 2004-2005).

En un primer intento de medición de las consecuencias de este consumo, y de acuerdo con Cáceres et al. (2006: 522) se pueden identificar tres grandes categorías: "1) los efectos crónicos sobre la salud como el daño a ciertos órganos o la aparición de enfermedades, 2) los efectos físicos que ocasiona directamente la sustancia en un periodo corto de tiempo, 3) los efectos sociales derivados del consumo, como el daño de las relaciones interpersonales, la pérdida del trabajo, la desintegración familiar entre otros". Sin lugar a dudas, las pérdidas y consecuencias de este comportamiento se reflejan en tremendos costes económicos, sanitarios, sociales y personales.

La Economía de la Salud ha tomado buena cuenta de estas cifras devastadoras. Desde el texto antecedente de Marshall (1920) en Principles of Economics sobre los efectos de adicción en la demanda, y el artículo de Becker y Murphy (1988), han surgido numerosas aportaciones científicas cuyo rasgo principal es la consideración de aspectos ya no estrictamente económicos, sino más propios de disciplinas como la Sociología o la Psicología.

De este modo, se ha podido constatar cómo determinados factores psico- 
sociales aumentan o disminuyen la probabilidad de consumo de drogas y sus consecuencias adversas. En este sentido, Cáceres et al. (2006) priorizan las siguientes variables de las que se dispone de evidencia empírica psicosocial: autoestima, alteraciones psicológicas, comportamientos perturbadores, bajo autocontrol, déficit de habilidades sociales, habilidades emocionales, habilidades de afrontamiento, preconceptos y valoración de las drogas, espiritualidad, maltrato, disfunción familiar, relaciones con personas consumidoras, insatisfacción con las relaciones interpersonales.

Arellánez-Hernández, Díaz-Negrete, Wagner-Echeagaray y Pérez-Islas (2004) cargan las tintas en la "factura mental" que predice y ocasiona este consumo, proponiendo por ejemplo cómo la depresión aparece como factor antecedente, trastorno concurrente $\mathrm{o}$ efecto derivado del propio uso de estas sustancias. A las mismas conclusiones se ha llegado con la asociación entre consumo indebido, estrés y tensión psicosocial (Unger et al., 1998).

En cierto sentido, el consumo de drogas representa una forma inadaptativa de afrontamiento, y en particular un medio para aliviar la ansiedad y la depresión, síntomas inequívocos de sufrimiento psicológico (Muesser, Drake \& Wallach, 1998). En el estudio con muestras de adolescentes se ha encontrado que aquellos que no consumen drogas disponen de un repertorio más amplio de comportamientos de afrontamiento de situaciones potencialmente estresantes, en comparación con los consumidores (González, 1992).

No obstante, el consumo de drogas mediatizado por estos factores puede adoptar diferentes parámetros, presentándose de modo experimental, social, regular, intenso o compulsivo (World Health Organization, 2004). En los jóvenes, la secuencia de dependencia obedecerá a las características del consumo de la droga (tipo, cantidad y frecuencia), así como a las expectativas y los factores de vulnerabilidad personal, y las condiciones familiares y sociales en que se realiza el consumo (Cáceres et al., 2006).

La experimentación, consumo abusivo y abuso constituyen las principales etapas del consumo de drogas en la adolescencia (Graña, Muñoz \& Delgado, 2000). Cuanto más temprano se empiece a consumir las distintas sustancias adictivas es más probable que se sigan consumiendo de adultos y que se prueben incluso otras drogas más duras (Swadi, 1988).

Se dispone de suficiente evidencia empírica para el caso de los adolescentes norteamericanos que refleja un progresivo deterioro del estado de su salud desde principios de la década de 1980 . Teniendo en cuenta que el estado de salud es un proceso acumulativo, los problemas de salud en la juventud repercutirán negativamente en su estado de salud cuando sean adultos (Lakda- 
walla, Goldman \& Bhattacharya, 2001). Posponer la edad en la que, por ejemplo, los jóvenes comienzan a fumar mejorará significativamente la salud de los adultos a largo plazo (Glied, 2002).

Por ello, recientemente asistimos a una creciente preocupación en la identificación de aquellos factores que determinan la experimentación de las drogas en la adolescencia. Estos factores de riesgo por lo general se agregan en tres grupos: predisposición personal, factores ambientales (familia y amigos) y sucesos (Swadi, 1999).

Las personas de nuestros círculos sociales influyen de manera considerable en las etapas iniciales de experimentación y consumo abusivo de drogas. No solo determinadas conductas deben ser definidas como perniciosas, a menudo, los entornos familiares y comunitarios pueden ser también considerados de alto riesgo (González, Valdez, Domínguez, Palomar \& González, 2008). Los jóvenes españoles consideran las drogas como algo cercano, y lo que es peor, como algo que pueden controlar, lo que les conduce a no sentirse tan vulnerables a sus efectos como generaciones anteriores. Los consumidores de drogas ya no son considerados delincuentes, sino personas normales y cercanas (Rodríguez San Julián et al., 2005; Shiner \& Newburn, 1997).

Sin embargo, todavía hoy la consecuencia de que el consumo de drogas se siga percibiendo como una actividad "desviada" por la sociedad, es que muchos padres consideran que sus hijos no caerán en la tentación de probar drogas ilegales como las drogas recreativas.

De hecho, muchas investigaciones denuncian que la influencia de los padres es la herramienta más infrautilizada en la prevención de la drogadicción en la adolescencia (Califano, 2000; Jenkins \& Zunguze, 1998; Office of National Drug Control Policy, 1997; Resnick et al., 1997). Esta influencia se puede ejercer de diversas maneras, como la transmisión de valores sobre las drogas, la comunicación de estos valores o el control sobre cómo los jóvenes pasan su tiempo libre, entre otras acciones.

\section{BASE DE DATOS Y PRIMEROS ESTADÍSTICOS DESCRIPTIVOS}

De la Encuesta Sobre Drogas a la Población Escolar (2006) hemos extraído un total de 26.446 observaciones, las cuales corresponden a estudiantes con edades comprendidas entre los 14 y 18 años. Dicha encuesta es realizada por el Ministerio Español de Sanidad y Consumo desde 1994 y es representativa de los escolares españoles (MSC, 2006).

La evolución temporal de las drogas de diseño se manifiesta estable con un ligero repunte positivo en el 2000, el cual fue amortiguándose en años posteriores. Entre los estudiantes que han 
consumido drogas de diseño, la mayoría las prueban por primera vez a partir de los 16 años. La evolución de la edad de inicio tampoco revela que se empiecen a consumir antes. Si el dato positivo es que no hay evidencias de que el consumo de drogas de diseño se dispare en los próximos años, tampoco hay evidencias de que su consumo se reduzca (véanse los gráficos 1 y 2).

Las drogas de diseño son de diversos tipos. Entre las distintas opciones, las más consumidas son las anfetaminas; un $3,4 \%$ de los estudiantes españoles de secundaria las han consumido alguna vez. A las anfetaminas le siguen en orden de importancia el éxtasis (3,2\%), los alucinógenos $(2,8 \%)$ y el éxtasis líquido $(1,2 \%)$. El tipo de droga es importante porque su composición determina la extensión de las consecuencias negativas de su consumo, pero no es el único factor que se debe tener en cuenta. La frecuencia de consumo, si se combina con otras drogas, también juegan un rol significativo en el bienestar de los jóvenes. El 6,8\% de los estudiantes de secundaria han consumido drogas de diseño alguna vez, y por lo general no suelen probar los distintos tipos de estas.

Como cabe esperar, la frecuencia de consumo cae al considerar intervalos de tiempo más cercanos. El 4,9\% de los estudiantes consumieron estas drogas en los últimos 12 meses y un 2,6\% en los últimos 30 días.

Aunque los porcentajes caen de manera considerable, no hay que menos- preciar el hecho de que la mitad de los estudiantes que han probado drogas de diseño alguna vez las han consumido en los últimos 12 meses y un tercio en los últimos 30 días. Por lo tanto, hay signos de que se trata de una droga bastante adictiva, tal que la probabilidad de su consumo una vez probada es alta. En cuanto al pluriconsumo, cada droga tiene su mitología; en el caso de las drogas de síntesis, se divulgó la idea de que sus consumidores se abstenían de probar otras sustancias, limitándose a acompañar sus pastillas con cantidades generosas de agua. Sin embargo, observamos que entre los consumidores de drogas de diseño, el número de los que consumen bebidas alcohólicas semanalmente es tres veces superior al que no lo hace, y el número de los que fuman diariamente es el doble de los que no lo hacen. Por lo tanto, el consumo de drogas de diseño también está ligado al consumo de alcohol y tabaco (véanse las tablas 1,2 y 3 ).

Los motivos que conducen a los jóvenes a demandar drogas de diseño son diversos. El motivo principal es para pasarlo bien $(54,4 \%)$; para sentir nuevas sensaciones las consume el 15,9\%, y para mejorar las relaciones sociales el $2,4 \%$. No deja de ser preocupante que un $15,9 \%$ de los consumidores lo haga sin ningún motivo en especial (véase la tabla 4). 


\section{Gráfico 1}

Evolución del consumo de drogas de diseño entre los jóvenes españoles (\%).

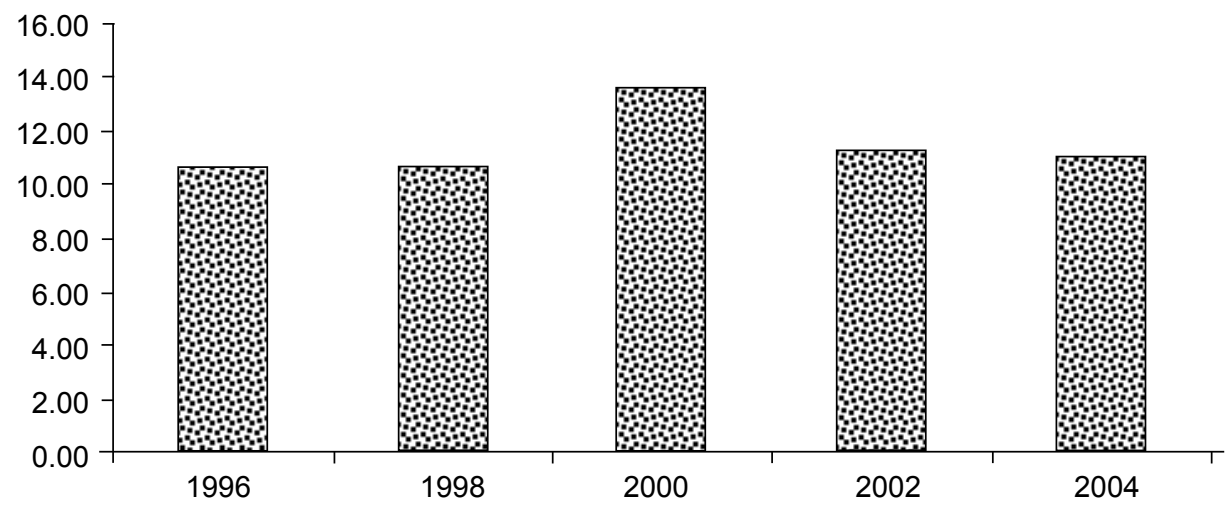

Gráfico 2

Evolución del consumo de drogas de diseño según edad y año de nacimiento (\%).

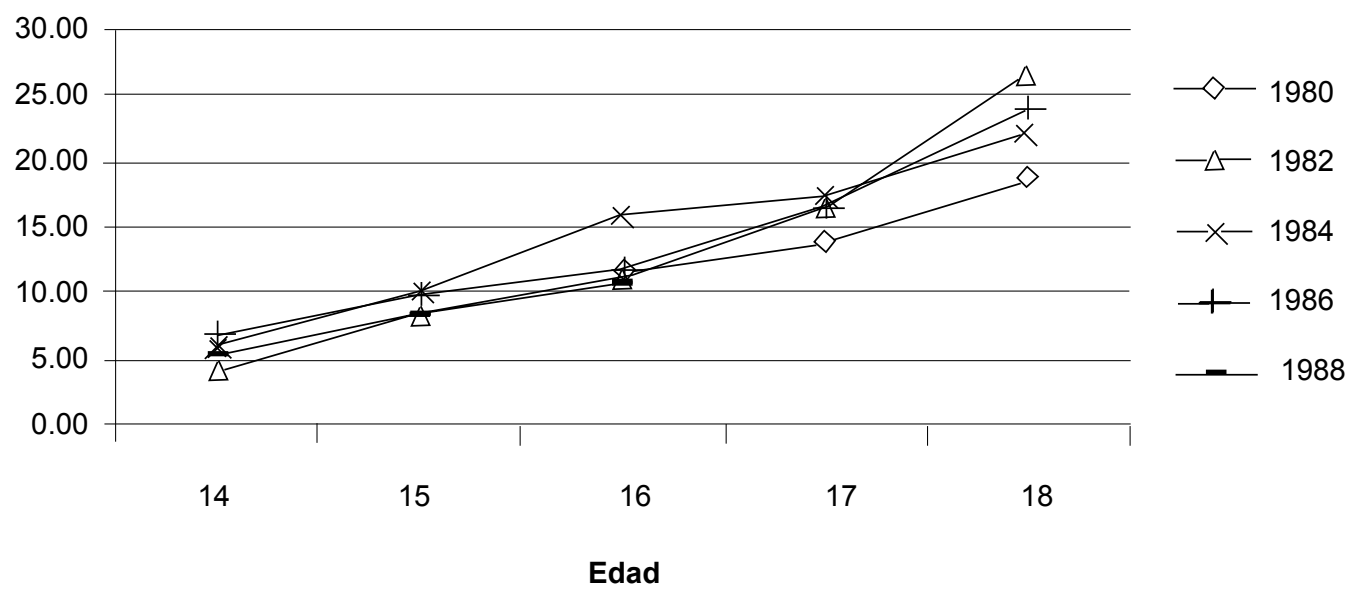


Tabla 1

Consumo de drogas de diseño alguna vez

\begin{tabular}{lcccc}
\hline & GH/éxtasis líquido & Éxtasis & Speed/anfetaminas & Alucinógenos \\
\hline Sí & 311 & 856 & 902 & 746 \\
$\%$ & $1,2 \%$ & $3,2 \%$ & $3,4 \%$ & $2,8 \%$ \\
Total & 26.446 & 26.445 & 26.443 & 26.442 \\
\hline
\end{tabular}

Fuente: Encuesta sobre Drogas a la Población Escolar 2006.

Tabla 2

Prevalecía consumo drogas de diseño

\begin{tabular}{lrrrrrr}
\hline & \multicolumn{2}{c}{ Alguna vez } & \multicolumn{2}{c}{ 12 meses } & \multicolumn{2}{c}{ 30 días } \\
\hline No & 24.582 & $93,2 \%$ & 25.141 & $95,1 \%$ & 25.746 & $97,3 \%$ \\
Sí & 1.806 & $6,8 \%$ & 1298 & $4,9 \%$ & 690 & $2,6 \%$ \\
Total & 26.388 & $100.0 \%$ & 26.439 & $100.0 \%$ & 26.436 & $100.0 \%$ \\
\hline
\end{tabular}

Fuente: Encuesta sobre Drogas a la Población Escolar 2006.

Tabla 3

Pluriconsumo de drogas

\begin{tabular}{lcccc}
\hline \multirow{2}{*}{ Drogas de diseño } & \multicolumn{2}{c}{ Alcohol semanalmente } & \multicolumn{2}{c}{ Tabaco diariamente } \\
\cline { 2 - 5 } & No & Si & No & Si \\
\hline No & 18.051 & 6.343 & 19.860 & 4.669 \\
Sí & 480 & 1.315 & 593 & 1210 \\
Total & 18.531 & 7.658 & 20.453 & 5.879 \\
\hline
\end{tabular}

Fuente: Encuesta sobre Drogas a la Población Escolar 2006.

Tabla 4

Motivos principales por los que se consume drogas de diseño

\begin{tabular}{lcr}
\hline Motivo & Observaciones & Frecuencia \\
\hline Por diversión & 375 & $54,4 \%$ \\
Para mejorar las relaciones sociales & 17 & $2,4 \%$ \\
Para sentir nuevas sensaciones & 110 & $15,9 \%$ \\
Por ningún motivo en especial & 186 & $27,1 \%$ \\
\hline Total & 690 & $100,0 \%$ \\
\hline
\end{tabular}

Fuente: Encuesta sobre Drogas Población Escolar 2000. Datos no disponibles en la encuesta del 2006. 
Tabla 5

Número de individuos con problemas derivados del consumo drogas de diseño

\begin{tabular}{lcccc}
\hline Problemas & $\begin{array}{c}\text { GH/éxtasis líquido } \\
(\mathbf{N} .=311)\end{array}$ & $\begin{array}{c}\text { Éxtasis } \\
\text { (N.= 856) }\end{array}$ & $\begin{array}{c}\text { Speed/anfetaminas } \\
(\mathbf{N} .=\mathbf{9 0 2})\end{array}$ & $\begin{array}{c}\text { Alucinógenos } \\
\text { (N.= 746) }\end{array}$ \\
\hline Accidentes con asistencia sanitaria & 18 & 25 & 35 & 34 \\
Frecuencia & $5,8 \%$ & $2,9 \%$ & $3,9 \%$ & $4,6 \%$ \\
Peleas & 16 & 40 & 92 & 37 \\
Frecuencia & $5,1 \%$ & $4,7 \%$ & $10,2 \%$ & $5,0 \%$ \\
Asistencia sanitaria & 9 & 14 & 23 & 14 \\
Frecuencia & $2,9 \%$ & $1,6 \%$ & $2,5 \%$ & $1,9 \%$ \\
Policía & 17 & 21 & 23 & 21 \\
Frecuencia & $5,5 \%$ & $2,5 \%$ & $2,5 \%$ & $2,8 \%$ \\
\hline
\end{tabular}

Fuente: Encuesta sobre Drogas a la Población Escolar 2006.

Tabla 6

Determinantes socio-demográficos del consumo drogas de diseño (Probit)

\begin{tabular}{|c|c|c|c|c|c|}
\hline \multirow[t]{2}{*}{ Variable } & \multicolumn{3}{|c|}{ Estimación Probit } & \multicolumn{2}{|c|}{ Primeros estadísticos } \\
\hline & Coeficiente & Mfx & St. Error & Media & Desv. Est. \\
\hline Hombre & $0,2184^{\star \star \star}$ & 0,0260 & 0,0294 & 0,4770 & 0,4994 \\
\hline Mujera & -- & -- & -- & 0,5230 & 0,5006 \\
\hline Edad & $0,1794^{* * *}$ & 0,0211 & 0,0111 & 15,706 & 1,3131 \\
\hline Convivepadres ${ }^{a}$ & -- & -- & -- & 0,8335 & 0,3725 \\
\hline Convivepadre & $0,2337^{\star * *}$ & 0,0316 & 0,0435 & 0,0158 & 0,1247 \\
\hline Convivemadre & 0,1457 & 0,0191 & 0,1175 & 0,1338 & 0,3405 \\
\hline Noconvivepadres & $0,2137^{* *}$ & 0,0295 & 0,0994 & 0,0158 & 0,1247 \\
\hline Madretrabaja ${ }^{a}$ & -- & -- & -- & 0,6099 & 0,4877 \\
\hline Madreamadecasa & $-0,0768^{* * *}$ & $-0,0088$ & 0,0333 & 0,3215 & 0,4670 \\
\hline Madreparada & $-0,0607$ & $-0,0068$ & 0,0979 & 0,0263 & 0,1602 \\
\hline Madreotro & 0,1093 & 0,0139 & 0,0844 & 0,0421 & 0,2009 \\
\hline Padretrabaja ${ }^{a}$ & -- & -- & -- & 0,8684 & 0,3380 \\
\hline Padreparado & $-0,0375$ & $-0,0043$ & 0,1150 & 0,0170 & 0,1294 \\
\hline Padreotro & 0,0397 & 0,0048 & 0,0528 & 0,1145 & 0,3184 \\
\hline Madreuniversidad & 0,0118 & 0,0014 & 0,0751 & 0,2629 & 0,4402 \\
\hline Madresecundaria & 0,0206 & 0,0024 & 0,0686 & 0,4205 & 0,4936 \\
\hline Madreprimaria & 0,0181 & 0,0021 & 0,0681 & 0,2496 & 0,4328 \\
\hline Madresinestudios ${ }^{a}$ & - & -- & -- & 0,0668 & 0,2497 \\
\hline Padreuniversidad & 0,0779 & 0,0094 & 0,0691 & 0,2963 & 0,4566 \\
\hline Padresecundaria & $-0,0152$ & $-0,0018$ & 0,0644 & 0,3988 & 0,4897 \\
\hline Padreprimaria & 0,0063 & 0,0007 & 0,0645 & 0,2304 & 0,4211 \\
\hline Ddinerosemanal & $0,2285^{\star \star *}$ & 0,0269 & 0,0151 & $35,3753^{b}$ & $53,7030^{b}$ \\
\hline Constante & $-346,0619^{\star * *}$ & -- & 21,3363 & -- & -- \\
\hline
\end{tabular}

a Variables tomadas como referencia.

b Variable original, sin logaritmos neperianos.

${ }^{* *} p<.01,{ }^{* * *} p<.001$ 
En contraposición, los problemas derivados de su consumo son también numerosos. El éxtasis líquido se presenta como el tipo de droga de síntesis con mayores externalidades negativas. El 5\% de los consumidores de éxtasis líquido reconocen haber recibido asistencia sanitaria, haber participado en peleas y haber tenido problemas con la policía derivados de su consumo. También destacamos que el 10,2\% de los consumidores de anfetaminas han participado en peleas tras haber consumido esta sustancia (véase la tabla 5).

\section{Determinantes del CONSUMO}

En esta sección presentamos los resultados de un modelo de selección probit, donde la variable a estimar es la probabilidad de haber consumido drogas de síntesis alguna vez. Las variables explicativas hacen referencia a las características sociodemográficas de los estudiantes españoles de secundaria (véase la tabla 6).

La muestra presenta un número similar de hombres y mujeres, teniendo los varones una probabilidad superior de haber consumido drogas de síntesis. La edad media de los estudiantes de la muestra es de 15,7 años, y como nos indica el gráfico 2, a mayor edad, mayor probabilidad de haber consumido drogas de diseño, tal que cada año incrementa dicha probabilidad en un $2,1 \%$.

La mayor parte de los estudiantes conviven con ambos padres (83,3\%), y la mayoría de los que no lo hacen conviven con la madre $(13,3 \%)$. La convivencia con ambos padres reduce el riesgo de que los estudiantes consuman drogas de diseño, y en caso de ausencia de uno de los progenitores se siente en mayor grado la ausencia de la madre.

Otra variable sociodemográfica con impacto en la decisión del estudiante de consumir drogas de síntesis es la situación laboral de la madre, de manera que los estudiantes cuyas madres son amas de casa tienen una probabilidad menor de consumir sustancias de síntesis con respecto a los estudiantes cuya madre trabaja. El impacto de dicha variable, aunque estadísticamente significativo, es reducido en magnitud (no alcanza el 1\%). Lo significativo es que el $32,1 \%$ de las madres de los estudiantes de secundaria son amas de casa.

De hecho, ni la situación laboral ni el nivel educativo de los padres tienen un reflejo claro sobre el consumo de drogas de diseño de sus hijos. En esta investigación nos hemos centrado en un parámetro básico del estudio de la familia: su composición sociodemográfica. La literatura científica psicosocial nos aporta numerosos ejemplos sobre la asociación entre dinámica, estructura familiar y consumo de drogas. Arellánez-Hernández et al. (2004: 56) nos describen la siguiente "radiografía": las estrategias familiares de adaptación a su entorno, el clima emocional, la capacidad para equilibrar la proximidad y la distancia en las relaciones 
intrafamiliares y la formación de fronteras generacionales funcionales cumplen un papel fundamental en la definición de condiciones de riesgo o protectoras. Las familias de consumidores de drogas suelen ser desagregadas $\mathrm{o}$, por el contrario, con pautas cohesivas que dificultan la individuación; con alta inconsistencia en el manejo de la autoridad, pautas negativas de modelamiento y de comunicación, límites difusos o rígidos y una marcada rigidez ante las presiones ejercidas por el medio o derivadas de los cambios relacionados con el ciclo de vida familiar.

El consumo de drogas de diseño se relaciona con un entorno familiar con unas determinadas coordenadas demográficas y psicosociales. En la muestra analizada hemos ampliado la consideración de este entorno a los condicionantes socioeconómicos que suponen su consumo, pero dado que las drogas de diseño son ilegales, no existe un registro fiable sobre su precio en el mercado negro.

La única variable monetaria de que disponemos para perfilar la demanda de estas sustancias por parte de la población escolar es su dinero disponible. En España pueden trabajar legalmente los jóvenes que al menos hayan cumplido los 16 años, por lo que la mayor parte de los estudiantes de secundaria solo pueden gastar aquel dinero que los padres les asignan semanalmente. Esta asignación semanal depende de la capacidad adquisitiva del hogar, pero también del grado de control que los padres quieran ejercer. Los padres que se preocupen de que sus hijos adquieran productos nocivos les dotarán de menos dinero. De hecho observamos que los estudiantes con mayor capacidad adquisitiva tienen una mayor tendencia a consumir drogas de diseño, aunque dichos incrementos se reducen a medida que aumenta el presupuesto. Los estudiantes de secundaria disponen en media de 35 euros semanales, aunque dicha asignación dista de ser homogénea para todo estudiante, pues la desviación estándar indica que hay estudiantes que reciben asignaciones superiores e inferiores.

Estos datos se pueden complementar con la información obtenida en el estudio de Fernández et al. (2003) sobre el consumo de drogas de síntesis en estudiantes de secundaria del Principado de Asturias (España) sobre la fuente de obtención del dinero y el lugar de adquisición. Teniendo en cuenta que el cuestionario permitía más de una respuesta, observamos que la gran mayoría de la muestra obtiene el dinero para adquirir pastillas a través de la paga familiar $(76,2 \%)$, trapicheos $(30,5 \%)$, trabajo $(17,4 \%)$, pequeños robos $(8,4 \%)$. El lugar de adquisición de las pastillas es: la calle $(59,3 \%)$, discotecas $(55,6 \%)$, bares $(32,8 \%)$, centro escolar $(5 \%)$. Las personas que las suministran son: los amigos (76,3\%), pequeños vendedores $(45,7 \%)$, el novio/a $(6,2 \%)$. El lugar de consumo: discote- 
cas $(87,9 \%)$, bares $(63,5 \%)$, naves, carpas, alrededores $(15,8 \%)$, calles $(15,6 \%)$ y en casa o en casa de amigos $(12,8 \%)$.

\section{ANÁLISIS Y CONCLUSIONES}

Aunque no existen evidencias de que el consumo de drogas de diseño aumente en los adolescentes españoles, se mantiene preocupantemente alto. Casi un $7 \%$ de los estudiantes de secundaria han probado dichas sustancias, la mitad de los que experimentaron con estas drogas lo hicieron en los últimos 12 meses y un tercio en los últimos 30 días. El carácter adictivo de estas sustancias agrava el hecho de que ya el consumo de una sola dosis entraña graves peligros para la salud. Los adolescentes corren riesgos y los corren reiteradamente.

A esto se suma el hecho ya constatado en otros estudios, de que en la juventud española y europea se asocian a estas drogas con el pluriconsumo de fin de semana. El alcohol se mezcla con casi todas las sustancias, así como el cannabis y la cocaína (Irefrea, 2001). Al respecto, la investigación de Viña y Herrero (2004) sobre la covariación en el consumo de drogas en población universitaria establece tres patrones: el primero de "sustancias duras", compuesto por el consumo de anfetaminas, cocaína, heroína, y otras sustancias. En el segundo factor o "drogas sociales" saturan las variables de consumo de tabaco, canna- bis, alcohol y cocaína (esta en menor grado que en el primer factor). Un tercer factor, constituido por el consumo de tranquilizantes, aparece más encaminado a la solución de problemas que a la búsqueda de sensaciones.

La mitad de los consumidores de las drogas de diseño lo hacen para pasarlo bien, pero sorprende que para un $15 \%$ no haya ningún motivo en especial. Los estudiantes son conscientes de que esta forma de diversión tiene costes adicionales al precio del producto, pues una gran parte de los consumidores reconocen haber padecido problemas de salud y sociales derivados de su consumo.

Las tendencias de consumo que apuntan estos datos son coherentes con la investigación de Cáceres et al. (2006) en una muestra de estudiantes universitarios colombianos, cuyos resultados indican que "Los factores psicosociales y los factores psicológicos evaluados, de manera global, están relacionados significativamente con el consumo de todas las sustancias legales e ilegales [...]" (p. 526). Entre los factores psicosociales se consideró, por ejemplo, la disfunción familiar, el maltrato, el déficit de habilidades sociales; y para las variables psicológicas: los comportamientos perturbados, las habilidades de autocontrol, los preconceptos y la valoración de las drogas.

En el estudio de Fernández et al. (2003) los jóvenes consumidores de drogas de diseño manifestaron tener peores relaciones familiares y resulta- 
dos académicos que los nos consumidores, y en general los amigos y la familia de estos estudiantes consumían a su vez más drogas legales e ilegales que los amigos y la familia de los no consumidores.

Una dirección común de estos resultados no apuntan exactamente hacia el hedonismo de nuestros jóvenes, sino a que estos consumos se intensifican conforme aumenta la exposición a situaciones y a hechos estresantes. Arellanez-Hernández et al. (2004) se refieren a diferentes tipos de problemas (por ejemplo, los relativos al ajuste escolar y a situaciones disruptivas del núcleo familiar), y lo que es más importante, estas diferencias se producen en el hecho objetivo pero sobre todo en la intensidad del efecto subjetivo producido por la exposición a factores estresantes. Lógicamente, el resultado es circular, porque el consumo de drogas de diseño también puede contribuir al empobrecimiento de los recursos de ajuste.

Estos resultados sugieren que aunque las campañas informativas sobre drogas sean efectivas, para ser eficientes deberían ir acompañadas tanto de estrategias que incrementen la percepción de riesgo de consumo de estas sustancias entre la población de riesgo como de estrategias que potencien alternativas de ocio constructivas (deporte, música o lectura, entre otras) y expectativas personales y laborales futuras (vivienda y trabajo) y una educación asertiva y positiva en las habilidades socia- les, emocionales, comunicativas y de afrontamiento.

La institución escolar y el sistema sanitario no son los únicos responsables en estas cuestiones; el núcleo familiar cumple también un papel fundamental en el bienestar de los adolescentes. La clase social (nivel laboral y educativo de los padres) no ejerce una influencia clara y contundente en una dirección, pero sí la cohesión familiar. Los estudiantes que viven en hogares con ambos padres tienen una menor probabilidad de experimentar con las drogas de diseño; pero lo que es más importante, aquellos estudiantes cuyas madres son amas de casa y los que disponen de menor asignación semanal tiene una menor tendencia a experimentar con las drogas. Ambas variables las identificamos como variables de control; no es que recomendemos que las mujeres deban ser amas de casa para garantizar el desarrollo de sus hijos, pero sí que aquellos jóvenes que son más controlados por sus padres tienen menos opciones de adoptar comportamientos de riesgo.

Compartimos con Cáceres et al. (2006) la necesidad de seguir investigando sobre los determinantes psicosociales de este consumo. Para prevenir necesitamos conocer en profundidad cómo se configura la percepción de riesgo y vulnerabilidad personal frente a las drogas de diseño, el manejo del tiempo de ocio, el desarrollo de habilidades sociales, de autocontrol, de 
afrontamiento, de manejo y seguimiento de alteraciones psicológicas y de los comportamientos y las emociones perturbadoras.

La diversidad de factores identificados confirma la conveniencia de desarrollar modelos multifactoriales y sistémicos para la comprensión del fenómeno (Arellanez-Hernández et al., 2004). Los resultados obtenidos en esta investigación coinciden con otros trabajos de integración del fenómeno en un marco multicausal y multidisciplinar (Bell, Forthun \& Sun, 2000).

\section{REFERENCIAS}

Aleixandre, R.; Valderrama, J. C. \& Cervera, G. (2000). Producción científica nacional e internacional en drogas de diseño (1988-1997). Adicciones, 12, 195-205.

Arellánez-Hernández, J. L.; Díaz-Negrete, D. B.; Wagner-Echeagaray, F. \& Pérez-Islas, V. (2004). Factores psicosociales asociados con el abuso y la dependencia de drogas entre adolescentes: análisis bivariados de un estudio de casos y controles. Salud Mental, 27, 54-64.

Becker, G. \& Murphy, K. (1988). A theory of rational addiction. Journal of Political Economy, 96, 675-700.

Bell, N.; Forthun, L. F. \& Sun, S. W. (2000). Attachment, adolescent competencies and substance abuse: Developmental considerations in the study of risk behaviors. Substance Use and Misuse, 35, 11771206.

Buela-Casal, G. \& Sierra, J. C. (2001). Manual de evaluación y tratamientos psicológicos. Madrid: Biblioteca Nueva.

Cáceres, D.; Salazar, I.; Varela, M. \& Tovar, J. (2006). Consumo de drogas en jóvenes universitarios y su relación de riesgo y protección con los factores psicosociales. Universitas Psychogical, 5, 521-534.

Califano, J. A. (2000). Winning the war on drugs: It's all in the family. America, 182, 6-8.

Fernández, J.; Álvarez, E.; Secades, R.; Jiménez, J. M.; Cañada, A.; Donate, I.; Fernández, J. R. \& Vallejo, G. (2003). Consumo de drogas de síntesis en estudiantes de secundaria del Principado de Asturias (España). Adicciones, 15, 31-37.

Glied, S. (2002). Youth tobacco control: reconciling theory and empirical evidence. Journal of Health Economics, 21, 117-135.

González, C. (1992). Estrés y respuestas de afrontamiento. Impacto sobre el estudio emocional en adolescentes. Tesis de Maestría. Universidad Nacional Autónoma de México.

González, I.; Valdez, J. L.; Domínguez, A.; Palomar, J. \& González, S. (2008). Características psicosocia- 
les en niños en contextos de riesgo y de no riesgo. Persona, 11, 91-108.

Graña, J. L.; Muñoz, M. J. \& Delgado, S. (2000). Investigación sobre el consumo de drogas en la adolescencia de Majadahonda: factores de riesgo y de protección. Madrid: Ayuntamiento de Majadahonda.

Hussey, D. L. \& Singer, M. I. (1997). Adolescents: Direct practice. En R. L. Edwards (Ed.). Encyclopedia of social work. Washington, DC: NASW Press.

Irefrea (2001). Salir de marcha y consumo de dogas. <http://www. mir.es/pnd/doc/observat/estudios/ir efrea/1Febrero2001>.

Jenkins, J. E. \& Zunguze, S. T. (1998). The relationship of family structure to adolescent drug use, peer affiliation, and perception of peer acceptance of drug use. Adolescence, 33, 811-822.

Lakdawalla, D.; Goldman, D. \& Battacharya, J. (2001). Are the young becoming more disabled? NBER Working Papers 8247, National Bureau of Economic research, Inc.

Marshall, A. (1920). Principles of economics. Londres: Mcmillan.

Ministerio de Sanidad y Política Social, MSC (2006). Encuesta Estatal sobre Uso de Drogas en Estudiantes de Enseñanzas Secundarias 2006. $<$ http://www.pnsd.msc.es/Categoria 2/observa/pdf/Estudes2006.pdf $>$.
Muesser, K. T.; Drake, R. E. \& Wallach, M. A. (1998). Dual diagnosis: Review of ethiological theories. Addictive Behaviors, 23, 717-734.

National Institute on Drug Abuse (2000). NIDA infofacts: Club drugs. <http://www.drugabuse.gov/ Infofax/clubdrugs.html>.

Office of National Drug Control Policy, UNODC (1997). National youth anti-drug media campaign: Communication strategy statement. Washington, DC: Office of National Drug Control Policy.

Office of National Drug Control Policy, UNODC (2004). Word Drug Report 2004. <http:www.unodc.org/unodc/ en/world_drug_report.html>.

Office of National Drug Control Policy, UNODC (2005). Word Drug Report 2005. <http:www.unodc. org/unodc/en/world drug report. html>.

Pérez, A.; Leal, F. J.; Jiménez, I.; Mesa, M. I.; Martínez, M. L. \& Pérez, R. (2007). Evolución del consumo de sustancias tóxicas en los adolescentes de una zona urbana (19972004). Atención primaria, 39 , 299-304.

Plan Nacional sobre Drogas (2001). Drogas: + información - riesgos. Madrid: Ministerio del Interior.

Pruitt, D. B. (1999). The American Academy of Child and Adolescent 
Psychiatry: Your adolescent: Emotional, behavioral, and cognitive development from early adolescence through the teen years. Nueva York: Harper Collins.

Resnick, M. D.; Bearman, P. S.; Blum, R. W.; Bauman, K. E.; Harris, K. M.; Jones, J.; Tabor, J.; Beuhring, T.; Sieving, R. E.; Shew, M.; Ireland, M.; Bearinger, L. H. \& Udry, J. R. (1997). Protecting adolescents from harm: Findings from the National Longitudinal Study on Adolescent Health. Journal of the American Medical Association, 278, 823832.

Rodríguez San Julián, E.; Megías Quirós, I.; Megías Valenzuela, E. \& Navarro Botella, J. (2005). La percepción social de los problemas de drogas en España, 2004. Madrid: FAD.

Shiner, M. \& Newburn, T. (1997). Definitely, maybe not? The normalisation of recreational drug use amongst young people. American Journal of Sociology, 511-529.
Swadi, H. (1988). Drug and substance use among a population of 3,333 London adolescents. British Journal of Addiction, 83, 935-942.

Unger, J. B.; Kipke, M. D.; Simon, T. R.; Jonson, C. J.; Montgomery, S. B. \& Iverson, E. (1998). Stress, doping and social support among homeless youth. Journal of Adolescence Research, 13 (2), 134-157.

U.S. Department of Health and Human Services (2003). Club drugs. $<\mathrm{http}$ ://www.girlpower.gov/girlarea /bodyfx/clubdrugs.htm>.

Viña, C. M. \& Herrero, M. (2004). El consumo de sustancias psicoactivas en estudiantes de Psicología de la Universidad de la Laguna. International Journal of Clinical and Health Psychology, 4, 521-536.

World Health Organization, WHO (2004). Global status report on alcohol 2004. <http://www.who.int/ substance_abuse/publications/alcohol/en/index.hmtl>. 